\title{
Side-View Operando Optical Microscopy Analysis of a Graphite Anode to Study Its Kinetic Hysteresis
}

\author{
Sujin Kang, Su Jeong Yeom, and Hyun-Wook Lee ${ }^{*[a]}$
}

\begin{abstract}
Operando analyses have provided several breakthroughs in the construction of high-performance materials and devices, including energy storage systems. However, despite the advances in electrode engineering, the formidable issues of lithium intercalation and deintercalation kinetics cannot be investigated by using planar observations. This study concerns side-view operando observation by optical microscopy of a graphite anode based on its color changes during electrochemical lithiation. Since the graphite color varies according to the optical energy gap during lithiation and delithiation, this technique can be used to study the corresponding charge-discharge kinetics. In addition, the cell configuration uses liquid electrolytes similar to commercial cells, allowing practical application. Furthermore, this side-view observation has shown that microscale spatial variations in rate and composition control the insertion and deinsertion, revealing the kinetics throughout the whole electrode. The results of this study could enhance the fundamental understanding of the kinetics of battery materials.
\end{abstract}

Studies over the past decade have revealed the importance of operando imaging techniques in the study of various electrochemical reactions. In particular, operando microscopy has contributed to understanding the lithiation and sodiation behaviors in batteries to discover new, high-performance electrode materials and identify the fundamental failure mechanisms that limit their cycle life. ${ }^{[1-3]}$ Operando spectroscopic techniques, such as X-ray diffractometry, ${ }_{1}^{[4-6]}$ Raman spectrosco$\mathrm{py}_{1}{ }^{[7-9]}$ FTIR spectroscopy, ${ }_{1}^{[10]}$ and NMR spectroscopy, ${ }^{[11,12]}$ have shed light on the formidable and unknown questions of electrochemical reactions. However, they have always been constrained by the averaging of information in the material bulk or surface. Therefore, to further understand the specific local structure of the surface or bulk of electrochemically reactive species, direct imaging is required as it offers exceptional opportunities to monitor the dynamic processes of various electrode materials at high spatial and temporal resolution.

[a] S. Kang, S. J. Yeom, Prof. H.-W. Lee

School of Energy and Chemical Engineering

Ulsan National Institute of Science and Technology (UNIST)

50 UNIST-gil, Ulju-gun, Ulsan 44919 (Republic of Korea)

E-mail:hyunwooklee@unist.ac.kr

(4) Supporting Information (including all experimental details) and the

(iD ORCID identification number(s) for the author(s) of this article can be found under:

https://doi.org/10.1002/cssc.201903289.

This publication is part of a joint Special Issue with Batteries \& Supercaps focusing on "2D Energy Storage Materials". Please visit the issue at http://doi.org/10.1002/cssc.v13.6.
Optical microscopy (OM) is particularly beneficial for the observation of physical transitions, with a convenient experimental setup. For example, it allows us to utilize an electrochemical cell configuration similar to a real battery, which consists of liquid electrolytes and conventional electrodes fabricated on $\mathrm{Cu}$ or Al current collectors. Basically, in both cases, the interactions between charge transfer and diffusion of lithium ions are governed by the active materials in the electrodes rather than by the electrolytes. ${ }^{[13-15]}$ In contrast, the configurations of many spectroscopic analyses and scanning and transmission electron microscopes obfuscate this underlying mechanism owing to relatively large electrolyte contents in their minuscule devices, which lead to electrolyte-dominant reactions during the operando analysis.

Graphite anodes are still the most promising candidates for commercial application in lithium-ion batteries (LIBs), but retaining their kinetics on repeated charge-discharge cycles is a scientific challenge. ${ }^{[16,17]}$ In particular, understanding their kinetics at the electrode level is crucial to satisfy the high demand for rapid-charging batteries with the current LIB systems. ${ }^{[18]}$ Previous studies have dictated the charge transfer kinetics of solvation/desolvation processes and Li bulk diffusion in graphite. ${ }^{[19-21]}$ As all the electrochemical reactions of graphite occur simultaneously, the kinetics of lithiation and delithiation should also be considered under dynamic conditions, which would lead to better comprehension and enable better design of the graphite electrodes. Therefore, the direct observation of graphite anodes has attracted much interest. However, despite the consequent extensive studies on operando imaging techniques, such analyses are mostly limited to a single particle or a particular position of the electrodes. ${ }^{[22]}$ In this regard, Harris et al. and Shi et al. investigated operando OM analysis of a graphite anode to determine lithium mass transport during charge and discharge reactions. Although the previous approaches well demonstrated a diffusion model of lithium intercalation, numerous issues remain to be addressed from the viewpoint of interparticle mechanisms during charge and discharge reactions. ${ }^{[23,24]}$

The wavelength range of visible light $(380-740 \mathrm{~nm})$ influences the analytical range of macroscale features inside a battery. Nevertheless, it also allows the observation of every change in the electrode color due to lithium transport, in relation to the visible light absorbed. Unlike electron beam-based microscopy, $\mathrm{OM}$ can differentiate colors and thus is a suitable technique to investigate the optical energy gaps of those electrodes, especially graphite anodes, which do not exhibit noticeable morphological changes during $\mathrm{Li}$ intercalation. Uhlmann et al. monitored $\mathrm{Li}$ intercalation in graphite flakes by in situ OM. ${ }^{[25]}$ They observed color transitions based on the state of charge 
(SOC) and also lithium dendrites during a cell short circuit at a high current density. Depending on the intercalation stage, the graphite color changed from black to gold, which coincided with the change in energy gap due to the variation of the lattice spacing. ${ }^{[26]}$ However, the most substantial phenomena should be perpendicularly aligned with the electrode surface. Current side-view operando microscopes allow the real-time analysis of micromorphological changes and color transition by optical energy gap transient of the side view of an electrode or its interface (Figure 1).

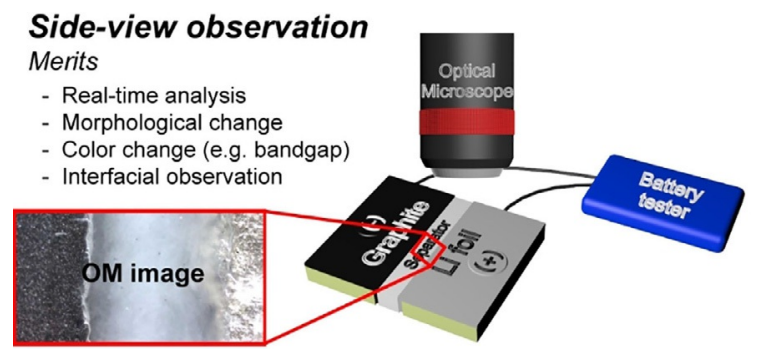

Figure 1. Schematic of a side-view operando optical microscopy (OM) cell during battery characterization. The working and counter (also reference) electrodes are made of graphite and metallic Li, respectively, and the scale bar in the OM image represents $500 \mu \mathrm{m}$. Operando OM allows the observation of the internal side-views of anodes, cathodes, electrolytes, and their interfaces.

Considering the merits of side-view operando microscopy, we ascertained new in-depth kinetics of graphite anodes according to lithiation and delithiation processes. We found that the Li deintercalation kinetics are much faster than the intercalation kinetics at the electrode level and the side-view observation showed that the graphite lithiation proceeds from the top interface with a separator to the bottom of the current collector, indicating different charge and discharge The most striking aspect of graphite intercalation species is probably their vivid colors in the charge and discharge states. Complementary colors are conventionally represented as color pairs on the opposite sides of the color wheel (Figure 2), which are also confirmed by the UV/Vis spectra of graphite intercalation species involving transitions among $\mathrm{sp}^{2}$ carbons. They can be used to

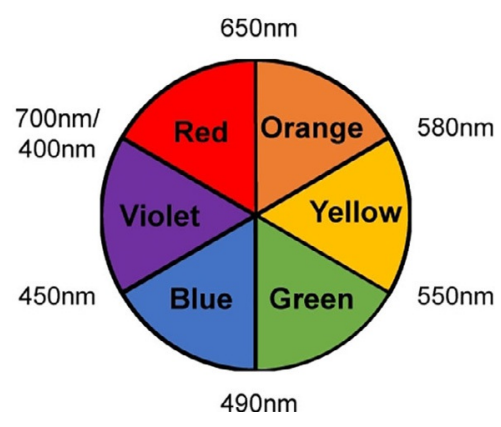

Figure 2. Color wheel with the wavelength ranges of each color; opposite colors in the wheel are complementary colors. When some wavelength of incident light is absorbed, the color complementary to the absorbed color appears. understand the state wavelengths of these species. For example, when an entire visible spectrum passes through an intercalated graphite anode that absorbs green wavelengths (490$550 \mathrm{~nm}$ ), the color observed is red; as red and green are complementary colors, the former predominates when the wavelengths of the latter are subtracted from the beam.

Photographs of graphite electrodes after cell disassembling are shown in Figure 3, where the different electrode colors, depending on the SOC and depth of discharge (DOD), can be observed. When the SOC increases, the color changes from black to violet (at SOC 30), indicating that the lithiated graphite electrode absorbs the yellow wavelength. Likewise, in a SOC, the lithiated graphite electrode exhibits red color (at SOC 40) by absorbing the green wavelengths $(490-550 \mathrm{~nm})$. Finally, the electrode absorbs violet wavelengths $(400-450 \mathrm{~nm})$ and thus shows yellow color. To summarize, at the initial stage, the visible wavelengths absorbed by a low SOC graphite anode are in the low-energy region and, then, shift toward the higher energies of yellow, green, and violet wavelengths, resulting in the appearance of the complementary colors of violet, red, and yellow, respectively. However, the discharge behavior differs from the charge behavior in terms of color changes. At DOD 10 and 20, the color immediately turns from yellow to red, which means a prompt change in the absorbed wavelength range during discharge, unlike in the charging process where the electrode becomes yellow at SOC 50; this indicates different tendencies of wavelength absorption according to the charge state. Although the specific capacities are reversible during charge and discharge, the absorption ranges do not coincide with the SOC and DOD, suggesting the need for realtime analytics to understand the dynamic kinetics.

To analyze the optical properties of the lithiated graphite anode, we measured their optical reflectivity by UV/Vis spectroscopy. This parameter correlates the energy gaps of graphite and the lithiated states corresponding to the color changes; its trend is shown as the energy gap variation with SOC and DOD in Figure $4 \mathrm{a}$. The energy gap was determined by the Kubelka-Munk theory based on the measured optical reflectivity $^{[27,28]}$ (see the Supporting Information, Figure S1 and Table S1). When lithiation occurs, electrons prefer to stay on the $\pi$ bands of graphite rather than the $2 \mathrm{~s}$ states of $\mathrm{Li}$; thus, the Fermi level lies within these $\pi$ bands as well. ${ }^{[29,30]}$ As a consequence, the Fermi level of lithiated graphite becomes higher than the initial state (Figure $4 \mathrm{~b}$ ). By lithium insertion, the aschanged Fermi level generates free electrons in the conduction band, resulting in a metallic property of lithiated graphite. At the higher lithiated state, the DOS at the $\pi$ band increases, owing to a large number of electrons, influencing the rise of the interband energy at the $\pi-\pi$ band that is reflected in the graphite electrode color (Figure 4c). Therefore, the gap between interband energies becomes larger during lithiation since more electrons are delocalized in the $\pi$ band and absorbs the incident light corresponding to its complementary color (Figure 3).

Side-view operando OM allows better observation of the dynamic lithiation behavior of graphite electrodes and the corresponding color change. The visible light penetrates through 


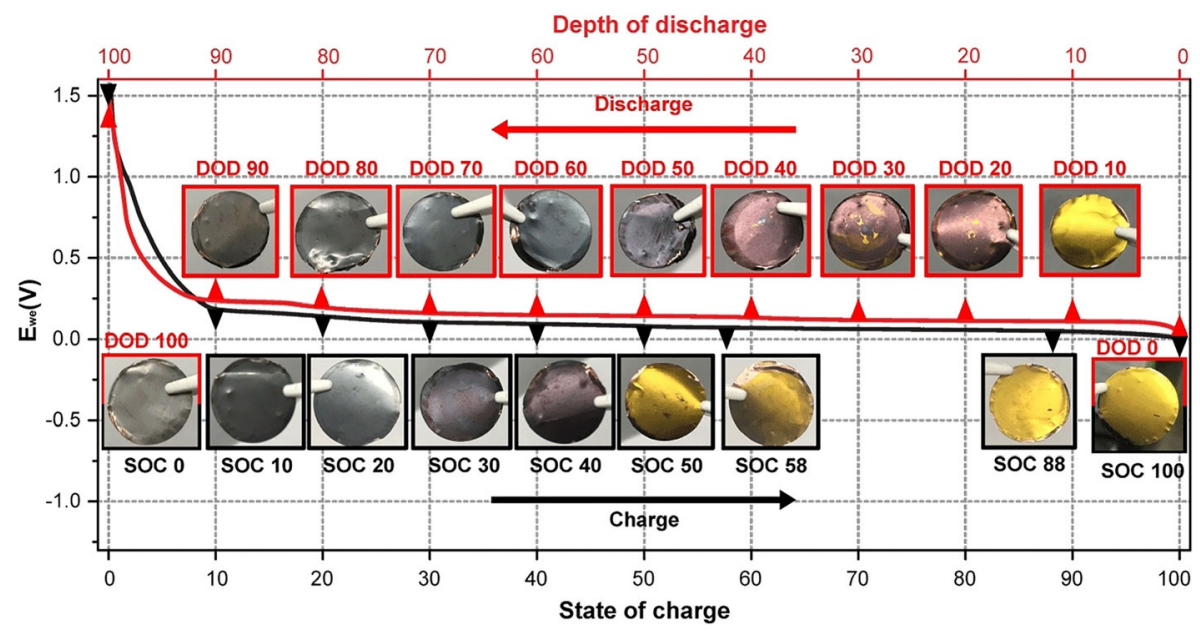

Figure 3. Ex situ photographs of a graphite electrode as a function of its state of charge (SOC) and depth of discharge (DOD). The black and red curves represent its charge and discharge behaviors, respectively. $E_{\mathrm{WE}}$ represents the electrochemical potential between the working and counter electrode.

a

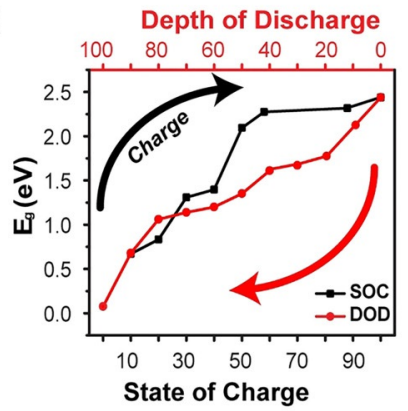

b

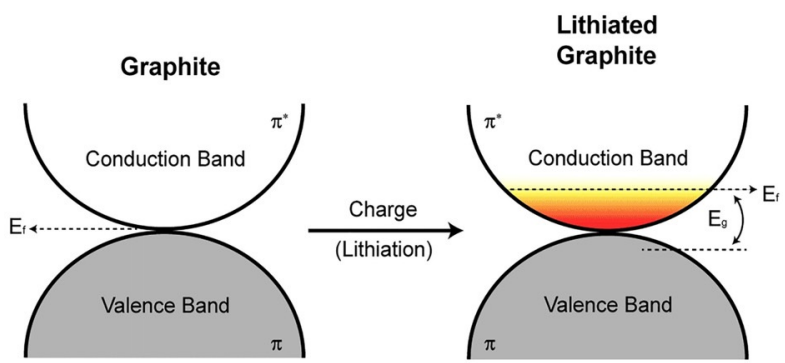

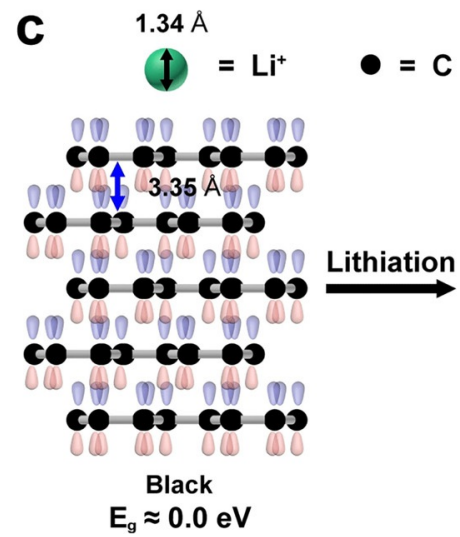
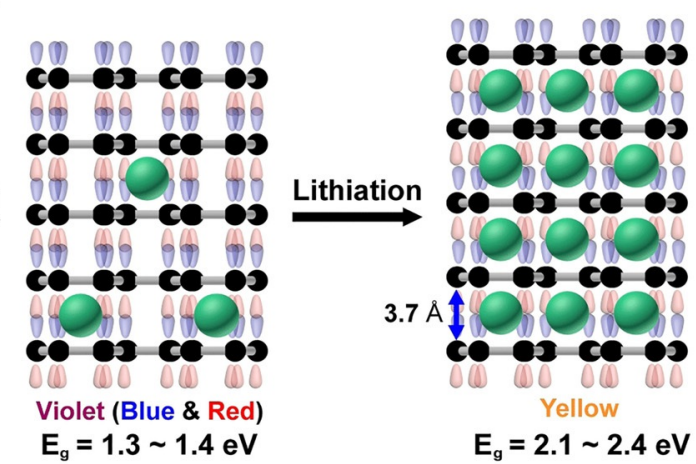

Figure 4. a) Interband energy gap $\left(E_{g}\right)$ of the graphite anode as a function of the state of charge and depth of discharge. b) Band gap structure at $\pi$ band of the graphite before (left) and after (right) lithiation. c) Schematic of the internal structure of the graphite anode during lithiation; Li ions are intercalated into the slabs between the graphite layers and the gap between them increases from 3.35 to $3.7 \AA$ because the p orbitals overlap with the other $p$ orbitals that change the weak bonding of lithiated graphite.

the top window of the side-by-side cell so that a lateral transition of the electrode can be monitored directly during lithiation. We simultaneously recorded the voltage profiles and OM images (for the morphology) of the lithiated graphite anode at a current density of $0.5 \mathrm{mAcm}^{-2}$; the time sequence of the images is shown in Figure $5 \mathrm{a}$. This cell can also be analyzed by Raman spectroscopy (Figure S2). At the beginning of lithiation, graphite starts to exhibit color variations to red and yellow from the separator/electrode interface. Then, the red-colored front moves slowly toward the current collector during the 1st charge stage (see the Supporting Information, Movie S1). In contrast, in the 1st discharge stage (delithiation; Movie S2), the reaction interface of graphite transforms simultaneously for a whole electrode range, although the discharge capacity is reversible as the charge capacity by the voltage profiles. The lithiated graphite distance is plotted against time in Figure $5 \mathrm{~b}$, 

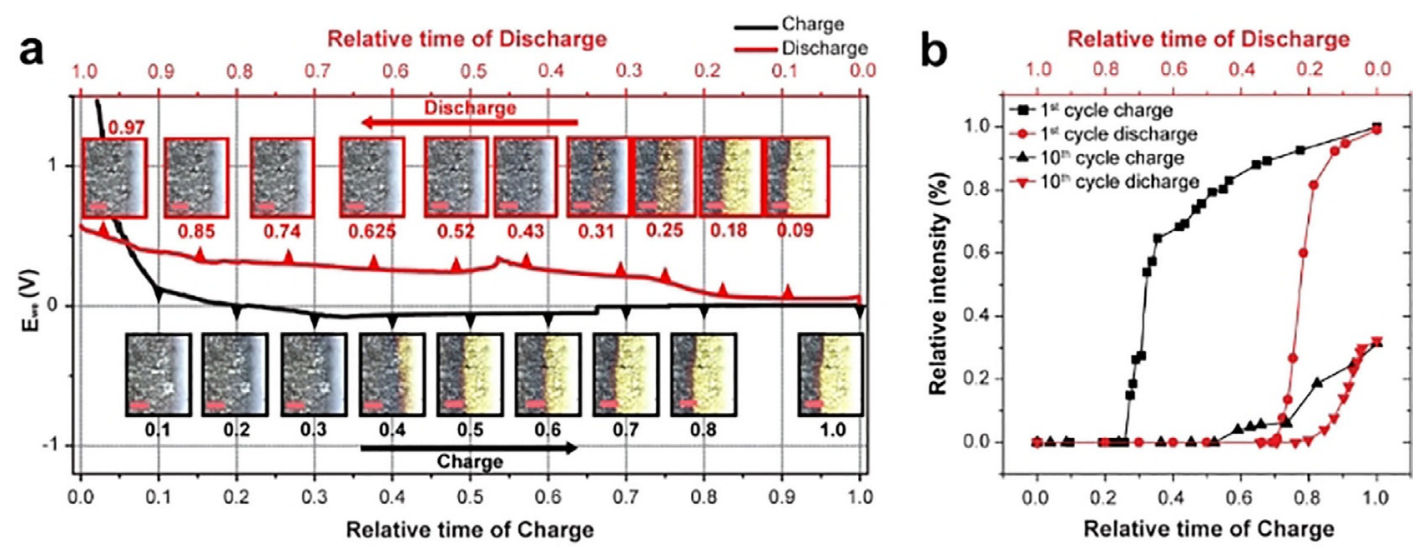

Figure 5. a) Side-view operando optical microscopy images (insets; scale bars represent $100 \mu \mathrm{m}$ ) of a graphite electrode during lithiation and its voltage profile for the 1st charge-discharge cycle. As the separator is on the right side of the images, the reaction at the anode/separator interface proceeds from the right to the left. b) Relative depth of charge as a function of the relative charge and discharge times for the 1st and 10th charge-discharge cycles. The sideview operando analysis implies hysteresis of charge and discharge behavior.

which clearly shows the charge and discharge hysteresis. This tendency towards different charge and discharge kinetics was not limited to the first charge-discharge cycle; we also observed it in the repeated cycles. The movies for the 10th cycles (Movies S3 and S4) show hysteretic behavior, suggesting that the diffusion of $\mathrm{Li}$ ions was dominated by the charge state. The lateral charge area increased gradually during lithiation, whereas the discharge slope dropped down, allowing rapid delithiation (Figure $5 \mathrm{~b}$ ). The slow increase of the charge slope indicates that lithiation proceeded gradually from the interface to the structure, which reiterates the significant challenge of realizing rapid charge batteries. If high current is applied to conventional graphite anodes, the kinetics of metallic Li formation surpasses that of $\mathrm{Li}$ intercalation, owing to limited reaction at the top graphite layer. Hence, metallic Li dendrites usually form on the top interface with a separator. Based on this phenomenon, which is related to the gap between graphite layers, the expansion of these layers would require high energy to widen the interfacial gaps and thus Li-ion intercalation would need more energy. In the delithiation case, this expansion energy term can be neglected, resulting in fast kinetics during Li deintercalation.

The ion intercalation mechanism of graphite is generally defined by the Rudroff and the Daumas-Herold models, which correspond to the stacking mechanism with the voltage profiles and the phase diagram. ${ }^{[31]}$ Based on the conventional mechanism, Li-ion intercalation into graphite should be dominant from the intraparticle viewpoint and hence this process should be physically limited by the rate of solid-state Li-ion diffusion. ${ }^{[32]}$ This is why an approach to rapid charging batteries with a graphite anode is generally to increase diffusion rates with a reduction in the diffusion path by minimizing the electrode dimensions. ${ }^{[33]}$ Our results have shown that spatial kinetic differences between charging and discharging account for the limited charge rate of a normal battery, suggesting the new kinetic model as an interparticle perspective observed by the top electrode surface to the bulk. In the discharge case, delithiation occurs almost simultaneously, which can be useful for the solid-state diffusion mechanism suggested in several prior reports. ${ }^{[34-36]}$

In summary, we have presented herein a side-view operando $\mathrm{OM}$ analysis of the lateral kinetics of electrochemical lithiation and delithiation in a graphite anode. We observed different charge and discharge kinetics dependent on the spatial distribution of the Li concentration within the electrode. The distribution gradient was relatively large in the transition region, which causes $\mathrm{Li}$ concentration distribution. The time variation of the distribution curves showed hysteresis and was affected by electrode phase transitions. Understanding the anomalous kinetics of Li-ion intercalation into graphitic anodes is crucial to optimizing this commercial and active material in terms of application, performance, lifetime, and safety. Although this observation is perhaps intuitively unsurprising, it contains several significant implications. Firstly, side-view operando OM allows a physical explanation for the optical energy gap changes exhibited by a wide range of batteries (such as metallic Li, volume-expanded alloys, and conversion anodes) and intercalation materials during the electrochemical reaction. ${ }^{[37-39]}$ This inconsistency has been traditionally attributed to the planar surface structures, which can differ significantly from the actual electrochemical interface areas. ${ }^{[25]}$ However, although this uncertainty about the active area undoubtedly contributes to variations reported for prior studies, the results presented herein indicate that lithium kinetics in any graphitic carbon depend critically on the size of the graphitized domains, as well as on their orientation relevant to the insertion and deinsertion flux. Secondly, our findings have immediate implications for the potential rational design of carbonaceous materials for high-rate anodes in LIBs. Despite the recent exploration of well-controlled structures and performance-oriented design of cathode materials, ${ }^{[40-42]}$ there are currently no guidelines to design carbon-based electrode architectures for LIBs, especially for high-power applications. Therefore, we believe that this in-depth study will open a new route to verify the kinetics of fast-charging research for satisfying both scientific and practical intrigue in the near future. 


\section{Acknowledgements}

This work was supported by Individual Basic Science \& Engineering Research Program (NRF-2019R1C1C1009324) and the Technology Development Program to Solve Climate Changes (2018M1A2A2063341) through the National Research Foundation of Korea funded by the Ministry of Science and ICT.

\section{Conflict of interest}

The authors declare no conflict of interest.

Keywords: batteries - kinetics - lithium - operando analysis optical microscopy

[1] H. W. Lee, Y. Li, Y. Cui, Curr. Opin. Chem. Eng. 2016, 12, 37-43.

[2] J. Sun, H. W. Lee, M. Pasta, H. Yuan, G. Zheng, Y. Sun, Y. Li, Y. Cui, Nat. Nanotechnol. 2015, 10, 980-985.

[3] S. W. Lee, H.-W. Lee, I. Ryu, W. D. Nix, H. Gao, Y. Cui, Nat. Commun. 2015, 6, 7533.

[4] J. R. Dahn, R. R. Haering, Solid State Commun. 1981, 40, 245-248.

[5] A. H. Whitehead, K. Edström, N. Rao, J. R. Owen, J. Power Sources 1996, $63,41-45$.

[6] A. S. Andersson, B. Kalska, L. Häggström, J. O. Thomas, Solid State lonics 2000, 130, $41-52$.

[7] T. Abe, Y. Mizutani, M. Asano, J. Electrochem. Soc. 1995, 142, 20-26.

[8] T. Itoh, H. Sato, T. Nishina, T. Matue, I. Uchida, J. Power Sources 1997, 68, $333-337$.

[9] K. Dokko, M. Mohamedi, N. Anzue, T. Itoh, I. Uchida, J. Mater. Chem. 2002, 12, 3688-3693.

[10] D. A. E. Goren, O. Chusid, J. Electrochem. Soc. 1991, 138, L6.

[11] M. Letellier, F. Chevallier, F. Béguin, E. Frackowiak, J. N. Rouzaud, J. Phys. Chem. Solids 2004, 65, 245-251.

[12] B. Key, R. Bhattacharyya, M. Morcrette, V. Seznéc, J. M. Tarascon, C. P. Grey, J. Am. Chem. Soc. 2009, 131, 9239-9249.

[13] Y. Wu, N. Liu, Chem 2018, 4, 438-465.

[14] N. Liu, G. Zhou, A. Yang, X. Yu, F. Shi, J. Sun, J. Zhang, B. Liu, C. L. Wu, X. Tao, Y. Sun, Y. Cui, S. Chu, Proc. Natl. Acad. Sci. USA 2019, 116, 765-770.

[15] Y. Wu, P. Huang, J. D. Howe, Y. Yan, J. Martinez, A. Marianchuk, Y. Zhang, H. Chen, N. Liu, Angew. Chem. Int. Ed. 2019, 58, 15228-15234; Angew. Chem. 2019, 131, 15372-15378.

[16] A. Doron, Z. Ella, C. Yaron, H. Teller, Solid State Ionics 2002, 148, $405-$ 416.

[17] V. Etacheri, R. Marom, R. Elazari, G. Salitra, D. Aurbach, Energy Environ. Sci. 2011, 4, 3243-3262.

[18] Y. Liu, Y. Zhu, Y. Cui, Nat. Energy 2019, 4, 540-550.
[19] K. Xu, Y. Lam, S. S. Zhang, T. R. Jow, T. B. Curtis, J. Phys. Chem. C 2007, $111,7411-7421$.

[20] Y. Yamada, Y. Iriyama, T. Abe, Z. Ogumi, Langmuir 2009, 25, 12766 12770.

[21] Y. Yamada, M. Yaegashi, T. Abe, A. Yamada, Chem. Commun. 2013, 49, $11194-11196$.

[22] J. Lim, Y. Li, D. H. Alsem, H. So, S. C. Lee, P. Bai, D. A. Cogswell, X. Liu, N. Jin, Y. S. Yu, N. J. Salmon, D. A. Shapiro, M. Z. Bazant, T. Tyliszczak, W. C. Chueh, Science 2016, 353, 566-571.

[23] S. Harris, A. Timmons, D. Baker, C. Monroe, Chem. Phys. Lett. 2010, 485, $265-274$.

[24] B. Shi, Y. Kang, H. Xie, H. Song, Q. Zhang, Electrochim. Acta 2018, 284 $142-148$.

[25] C. Uhlmann, J. Illig, M. Ender, R. Schuster, E. Ivers-Tiffée, J. Power Sources 2015, 279, 428-438.

[26] P. Delhaes, J. P. Manceau, D. Guerard, Synth. Met. 1980, 2, 277-284.

[27] P. Kubelka, F. Munk, Zeit. Für Tekn. Phys. 1931, 12, 593.

[28] J. H. Nobbs, Rev. Prog. Color. Relat. Top. 1985, 15, 66-75.

[29] N. A. W. Holzwarth, S. Rabii, L. A. Girifalco, Phys. Rev. B 1978, 18, $5190-$ 5205.

[30] N. A. W. Holzwarth, L. A. Girifalco, S. Rabii, Phys. Rev. B 1978, 18, $5206-$ 5216.

[31] C. Sole, N. E. Drewett, L. J. Hardwick, Faraday Discuss. 2014, 172, 223 237.

[32] M. J. Armstrong, C. O'Dwyer, W. J. Macklin, J. D. Holmes, Nano Res. 2014, 7, $1-62$.

[33] N. Kim, S. Chae, J. Ma, M. Ko, J. Cho, Nat. Commun. 2017, 8, 812.

[34] K. Persson, Y. Hinuma, Y. S. Meng, A. Van Der Ven, G. Ceder, Phys. Rev. B 2010, 82, 125416.

[35] K. Persson, V. A. Sethuraman, L. J. Hardwick, Y. Hinuma, Y. S. Meng, A. Van Der Ven, V. Srinivasan, R. Kostecki, G. Ceder, J. Phys. Chem. Lett. 2010, 1, 1176-1180.

[36] S. Thinius, M. M. Islam, P. Heitjans, T. Bredow, J. Phys. Chem. C 2014, 118, $2273-2280$.

[37] Y. Lee, J. Lee, J. Lee, K. Kim, A. Cha, S. Kang, T. Wi, S. J. Kang, H. W. Lee, N. S. Choi, ACS Appl. Mater. Interfaces 2018, 10, 15270-15280.

[38] W. Tang, X. Yin, S. Kang, Z. Chen, B. Tian, S. L. Teo, X. Wang, X. Chi, K. P. Loh, H. W. Lee, et al., Adv. Mater. 2018, 30, 1801745.

[39] S. A. Ahad, R. Pitchai, A. M. Beyene, S. H. Joo, D. K. Kim, H. W. Lee, ChemSusChem 2018, 11, 3402-3409.

[40] D. K. Kim, P. Muralidharan, H. W. Lee, R. Ruffo, Y. Yang, C. K. Chan, H. Peng, R. A. Huggins, Y. Cui, Nano Lett. 2008, 8, 3948-3952.

[41] H. W. Lee, P. Muralidharan, R. Ruffo, C. M. Mari, Y. Cui, D. K. Kim, Nano Lett. 2010, 10, 3852-3856.

[42] H. W. Lee, P. Muralidharan, C. M. Mari, R. Ruffo, D. K. Kim, J. Power Sources 2011, 196, 10712-10716.

Manuscript received: November 30, 2019

Revised manuscript received: January 24, 2020

Accepted manuscript online: January 27, 2020

Version of record online: March 5, 2020 